\title{
Pedido de ajuda em vítimas de violência no namoro: Revisão sistemática
}

\section{Help seeking in dating violence victims: A systematic review}

\section{Inês Pinheiro \\ Sónia Caridade $(1,2)$}

(1) Faculdade de Ciências Humanas e Sociais, Universidade Fernando Pessoa, Porto, Portugal

(1) Centro de Investigação em Ciências Sociais e do Comportamento, Observatório Permanente Violência e Crime, Portugal

Recebido: 12/06/2019; Revisto: 22/07/2019; Aceite: 20/10/2019.

https://doi.org/10.31211/rpics.2019.5.2.124

\section{Resumo}

Objetivo: Este estudo tem como objetivo sistematizar o conhecimento produzido sobre o pedido de ajuda em adolescentes e jovens adultos que experienciaram violência no namoro, através da identificação e análise dos estudos empíricos realizados neste âmbito. Métodos: Para tal adotámos os procedimentos para a realização de uma revisão sistemática dos estudos centrados nos comportamentos de pedido de ajuda em vítimas de violência no namoro. Resultados: Foram identificados 18 estudos com enfoque nos comportamentos de pedido de ajuda por parte dos/as adolescentes e jovens adultos vítimas de violência no namoro. A grande maioria dos estudos constatou que as vítimas revelam uma preferência pelo recurso a fontes de revelação informais, privilegiando os pares. As vítimas de sexo feminino surgiram como as que demonstraram uma maior tendência para a revelação do abuso íntimo, surgindo ainda como as expectadoras que prestam mais suporte e apoio às vítimas. Foram, ainda, identificadas múltiplas barreiras à revelação da experiência abusiva por parte dos/as participantes (e.g., legitimação da violência, medo de perder o/a parceiro/a, vergonha e autossuficiência para resolver a situação). Conclusões: Consideramos que a investigação neste domínio deverá procurar incluir outras amostras, estudos de continuidade, beneficiando de uma maior uniformização no tipo de instrumentos usados para efetuar a recolha de dados.

Palavras-Chave: Adolescentes; Jovens adultos; Pedido de ajuda; Revisão sistemática; Violência no Namoro.

\section{DI\&D | ISMT \\ rpics@ismt.pt}

https://rpics.ismt.pt

\author{
Sónia Caridade \\ Universidade Fernando Pessoa, \\ Praça 9 de abril, 349 \\ 4249-004, Porto, Portugal I \\ e-mail: soniac@ufp.edu.pt
}




\begin{abstract}
Objective: This study aims to systematize the knowledge about seeking help by adolescents and young adults experiencing dating violence through the identification and analysis of empirical studies in this field. Method: The procedures for conducting a systematic review of research on dating violence among adolescents and young people aged between 12-35, were adopted. Results: We identified 18 studies focusing on the behavior of requests for help from adolescents and young adults' victims of dating violence. The results show that dating violence victims expressed their preference for informal sources of disclosure in all studies, favoring friends. Female victims have shown a clear tendency to report violence, usually acting as spectators, and offering more help when compared to men. In this systematic review, it was also possible to identify the main barriers reported by adolescents and young people in accessing the sources of disclosure (i.e., legitimacy of violence, fear of losing the partner, shame, and lack of selfsufficiency to solve the situation). Conclusions: The conducted research can be applied to other samples, and continuity studies, benefiting from a uniform selection of the instruments used for data collection. The instruction of young people about the boundaries between normative and non-normative behavior in a dating relationship is essential, so that young people do not legitimize dating violence aggression, and may consequently seek help.
\end{abstract}

Keywords: Adolescent; Dating violence; Young adults; Seeking help; Systematic review.

\title{
Introdução
}

A violência no namoro tem sido amplamente reconhecida como um sério problema de saúde pública e social (Centers for Disease Control and Prevention, 2016; Organização Mundial de Saúde, 2016). Os diversos estudos produzidos no âmbito internacional (e.g., Hamby \& Turner, 2013; Jennings, Okeem, Piquero, Sellers, Theobald, \& Farrington, 2017; Straus, 2004) e no contexto português (e.g., Machado, Caridade, \& Martins, 2010; Neves, Cameira, Machado, Duarte, \& Machado, 2016; Santos, Caridade, \& Cardoso, 2019) apontam para indicadores de prevalência preocupantes e que atestam a necessidade premente de medidas preventivas e interventivas neste âmbito. Assim, no contexto português, um dos primeiros estudos conduzidos, e que inquiriu 4.665 jovens (Machado et al., 2010), apurou que um/a em cada quatro jovens já experienciou pelo menos um episódio de violência no namoro ao longo da vida. Num outro trabalho (Guerreiro et al., 2017), envolvendo 2.500 jovens com idades compreendidas entre os 12 e 18 anos de idade, $7 \%$ da amostra admitiu ter sido alvo deste tipo de abuso íntimo, pelo menos uma vez. No contexto internacional, estima-se que este fenómeno poderá situar-se entre os 15\% e os 80\% (Carter-Snell, 2015). Uma revisão sistemática (Jennings et al., 2017), envolvendo 169 estudos com jovens entre os 15 e os 30 anos, apurou estimativas de prevalência mais baixas entre os/as mais jovens ( $<10 \%)$ comparativamente com os/as mais velhos/as (entre 20 e 30\%), relatando as mulheres elevados indicadores de vitimação.

A adolescência assinala, geralmente, o período em que se iniciam as primeiras relações amorosas, sendo também, e não raras vezes, palco para a manifestação dos primeiros padrões de violência na intimidade. Os adolescentes podem ser confrontados com situações relacionais inesperadas, pautadas por comportamentos violentos e outras formas de coerção, face aos quais poderão ser levados a adotar uma postura de legitimação, percecionando tais atos como uma manifestação de amor e/ou ciúme (Caridade \& Machado, 2013). Tem sido igualmente documentado que a violência nas relações de intimidade poderá ter início na pré-adolescência e prolongar-se até à idade adulta (Caridade, 2011).

Os efeitos e implicações perniciosas da violência no namoro no funcionamento psicológico, físico e sexual dos adolescentes e jovens adultos têm sido igualmente documentado por diversos estudos internacionais (e.g., 
Carter-Snell, 2015; Exner-Cortens, Eckenrode, \& Rothman, 2013; Foshee, Reyes, Gottfredson, Chang, \& Ennett, 2013) e alguns nacionais (e.g., Caridade \& Barros, 2018; Santos et al., 2019). Tem sido defendido que muitas vítimas de violência no namoro têm mais probabilidades de sofrer sequelas ao nível da saúde física, psicológica e social (e.g., lesões, abuso de substâncias, depressão, isolamento social, infeções sexualmente transmissíveis, gravidezes não desejadas e baixo desempenho escolar) do que jovens que não vivenciam conflitos nos seus relacionamentos íntimos (Centers for Disease Control and Prevention, 2012; Dixe, Rodrigues, Freire, Rodrigues, Fernandes, \& Dias, 2010). Está documentada relutância dos jovens em procurar ajuda para a situação de violência de que são alvo (Soares, Lopes, \& Njaine, 2013), devido a diversos fatores (e.g., não reconhecimento do comportamento abusivo e da condição de vitimação; o receio de serem responsabilizados pela experiência abusiva ou de que a informação não permaneça em segredo; incapacidade dos/as adolescentes em identificarem os problemas para os quais precisam de ajuda; a ausência de informação e competências para procurar ajuda; a crença de que os/as profissionais não lhes poderão proporcionar a ajuda e apoio que necessitam) (Caridade, Pinheiro, \& Dinis, 2019). Essa relutância tende a acentuar a gravidade desses efeitos, tornando oculto o problema da violência (Hébert, Van Camp, Lavoie, Blais, \& Guerrier, 2015). Por sua vez, tal poderá colocar a vítima num maior risco de uma nova vitimização e de sofrer os efeitos negativos associados à violência no namoro, os quais poderão ser minorados a partir do momento em que os/as jovens/adolescentes encetam o pedido de ajuda (Caridade, 2018). Quando a divulgação ou o pedido de ajuda é efetivamente levado a cabo, verifica-se que a divulgação informal (e.g., junto de amigos, familiares, colegas, vizinhos) é priorizada em relação à divulgação formal (e.g., junto da polícia, instituições de apoio à vítima, professores) (Sabina \& Ho, 2014). A este respeito cabe ainda referir que o pedido de apoio formal versus informal tem sido alvo de algum debate. Se, por um lado, se considera que o pedido de apoio informal proporciona apoio e suporte emocional, não permite, porém, providenciar a informação necessária, as competências e os recursos existentes para lidar com esta problemática e eventuais preocupações com a saúde mental das vítimas (Hedge, Sianko, \& McDonell, 2017). Por outro lado, outros autores (e.g., Whitlock, Wyman, \& Moore, 2014) consideram que o pedido de apoio junto de certos contextos sociais (e.g., escola) incrementa a possibilidade de os/as adolescentes procurarem os serviços de apoio, minimizando, deste modo, o risco de suicídio.

A configuração do apoio e suporte social, seja informal ou formal, revela-se fundamental na gestão e cessação das práticas abusivas em que os/as adolescentes/jovens se encontram envolvidos/as e nas consequências/efeitos que possam advir para o seu bem-estar, algo que deverá ser atendido, promovido e potenciado nas diferentes políticas de prevenção/intervenção que possam emergir (Caridade, 2018).

Face à documentada importância desta matéria, com a presente revisão sistemática da literatura procurou-se sistematizar o conhecimento produzido em matéria de pedido de ajuda por parte dos/as adolescentes e jovens adultos/as que experienciaram algum tipo de violência no âmbito das suas relações amorosas. De forma mais concreta pretendeu-se identificar as principais fontes de revelação eleitas pelos/as jovens para denunciar a sua experiência abusiva e a sua eficácia percebida, bem como as barreiras inerentes ao pedido de ajuda.

\section{Método}

\section{Procedimentos de pesquisa}

A pesquisa para efeitos da revisão sistemática foi conduzida nas seguintes bases de dados eletrónicas: Complementary Index, B-on, Medline, Sage Journal, Scopus e Social Science Citation Index, entre fevereiro e abril de 2018. Foram identificadas as seguintes palavras-chave usadas nas combinações e/ou nas línguas 
portuguesa (Violência no Namoro, Jovens, Pedido de Ajuda e Vítimas); inglesa (Dating violence, Youth's; Help-seeking and Victims); e espanhola (Violencia de pareja, Jóvenes, Pedido de ayuda y Víctimas). Não foi colocada qualquer restrição em termos de períodos de publicação. Também foi realizada uma pesquisa no Google, utilizando a mesma combinação de palavras-chave nas diferentes línguas, a fim de integrar outros trabalhos de relevância sobre o assunto e publicações encontradas através do método bola de neve, que não apareceram nas bases de dados citadas anteriormente.

Critérios de inclusão e exclusão. Os critérios de inclusão definidos para a integração dos estudos na presente revisão sistemática foram: i) os estudos que incluíam nas suas amostras participantes que admitiram estar ou ter estado envolvidos em situações de violência no namoro; ii) estudos que utilizassem as seguintes metodologias: qualitativas, quantitativas ou mistas e iii) estudos centrados nos comportamentos efetivos de pedidos de ajuda formal e/ou informal. Neste sentido, os critérios de exclusão foram: i) artigos de revisão simples, estruturada ou sistemática da literatura; ii) estudos que explorassem apenas intenções ou presunções de pedido de ajuda e iii) estudos que contemplassem relacionamentos fora do contexto de namoro (e.g., casamento).

Seleção dos artigos. A pesquisa permitiu identificar um total de 248 artigos para análise. A primeira fase da análise consistiu na revisão do título, onde foram excluídos 73 artigos por não terem relação com o tema, surgirem em duplicado e estudos que apenas estavam focados nos agressores de violência no namoro. Os estudos revistos positivamente foram tidos em conta segundo a informação do seu resumo, na qual 150 artigos foram excluídos por não se tratarem de estudos empíricos, por serem estudos focados na violência nas relações familiares e/ou conjugais e, por último, pela amostra do estudo não ser constituída por vítimas de violência no namoro. Feita esta análise, apenas 26 artigos preencheram os critérios de seleção para revisão numa segunda fase (Figura 1).

Mediante uma leitura integral dos 26 artigos, chegou-se ao número final de 15 estudos que cumpriam todos os critérios pretendidos. Numa terceira fase, procedeu-se à análise das referências bibliográficas dos materiais selecionados, através da técnica de bola de neve, e identificaram-se 19 estudos relevantes para o tema, no entanto apenas 3 se revelaram passíveis de serem integrados nesta revisão sistemática. Neste sentido, chegou-se ao número de 18 artigos que preencheram todos os critérios de inclusão definidos anteriormente. A Figura 1 ilustra como se desenvolveu todo o processo da seleção dos artigos nas duas fases da presente revisão sistemática da literatura.

De modo a autenticar os estudos que devem ou não ser incluídos (de acordo com os critérios inicialmente definidos) na revisão sistemática, foi utilizada uma versão traduzida da checklist de qualidade The PRISMA Statement (Moher, Liberati, Tetzlaff, Altman, \& PRISMA Group, 2009). Esta checklist tem como objetivo apurar a cientificidade dos artigos e é constituída por treze tópicos de análise (título, resumo, introdução (2), métodos (6), resultados (2) e conclusão). A classificação de cada artigo processou-se através de uma escala de Likert de zero a dois, onde: 0 - "Não reportado/Não especificado" (o artigo em análise não apresenta informações acerca do item em questão); 1 - "Pouco claro/ Relatado" (o item foi mencionado, mas não pormenorizado); 2 - "Adequadamente feito" (o item em análise foi mencionado e explicado ao longo do artigo). A avaliação dos artigos foi imprescindível para que nesta revisão sistemática fossem apenas incluídos os estudos de qualidade respeitantes ao tema em estudo. Posteriormente, e para analisar o acordo interavaliadores, procedeu-se ao cálculo do coeficiente capa de Cohen, através do software de análise estatística Statistical Package for the Social Sciences (SPSS). Após o cálculo deste 
coeficiente, verificou-se que o valor obtido foi de 0,75 ( $p=0,05)$, o que representa um grau de concordância moderado/elevado entre as avaliadoras, assim como um nível moderado de confiabilidade.

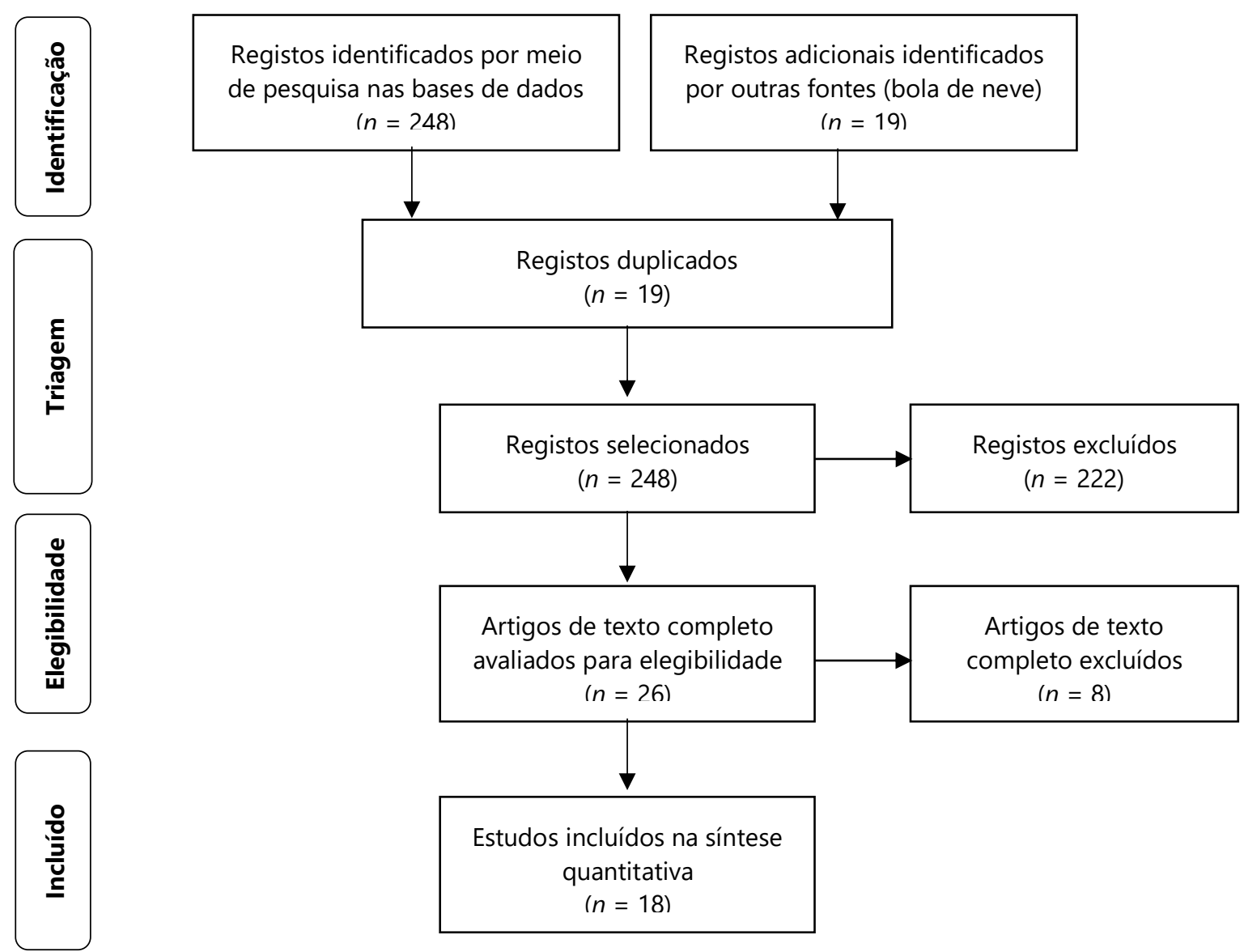

Figura 1. Fluxograma do processo de seleção dos artigos para a revisão sistemática.

Uma vez que desde o momento da identificação de artigos ao momento de extração dos mesmos decorreu um espaço temporal considerável (quatro meses), decidiu-se realizar um follow-up de identificação de novos artigos, tendo por base todos os procedimentos de pesquisa anteriormente descritos. Através deste follow-up identificaram-se seis artigos, no entanto apenas um foi integrado na revisão sistemática por apenas este corresponder aos critérios de inclusão definidos.

\section{Resultados}

Os dados dos 19 artigos foram extraídos e constam na Tabela 1, a qual apresenta informação detalhada sobre as principais características dos estudos, designadamente: características da amostra, instrumentos, e os principais resultados de cada um dos estudos de pesquisa em termos dos comportamentos relativos ao pedido de ajuda (diferenças de sexo, fontes utilizadas e ainda resposta percebida) e barreiras ao pedido de ajuda. 


\section{Tabela 1}

Síntese dos Estudos Considerados na Revisão Sistemática (continua)

\begin{tabular}{|c|c|c|c|c|c|c|c|c|}
\hline \multirow{3}{*}{ 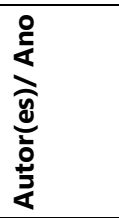 } & \multirow{3}{*}{$\begin{array}{l}\text { Objetivos do } \\
\text { estudo/País }\end{array}$} & \multicolumn{7}{|c|}{ Principais resultados } \\
\hline & & \multirow{2}{*}{$\begin{array}{c}\text { N, idade, sexo: } \\
M=\text { Mulheres } \\
H=\text { Homens }\end{array}$} & \multirow{2}{*}{ Medidas } & \multicolumn{4}{|c|}{ Comportamentos de pedido de ajuda } & \multirow{2}{*}{$\begin{array}{l}\text { Barreiras } \\
\text { à revelação }\end{array}$} \\
\hline & & & & $\%$ & $\begin{array}{c}\text { Diferenças } \\
\text { H e M }\end{array}$ & Fontes & Resposta percebida & \\
\hline 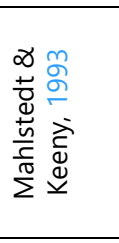 & $\begin{array}{l}\text { Comportamentos } \\
\text { de pedido de } \\
\text { ajuda } \\
\text { E.U.A. }\end{array}$ & $\begin{array}{c}130 \mathrm{M} \\
18-31 \text { anos }\end{array}$ & $\begin{array}{l}\text { Entrevista elaborada } \\
\text { para o efeito pelos } \\
\text { autores }\end{array}$ & 92,0 & - & $\begin{array}{c}80,0 \% \text { amigos } \\
47,0 \% \text { irmãs } \\
43,0 \% \text { irmãs } \\
25,0 \% \text { profissionais, } \\
\text { professores e } \\
\text { psicólogos } \\
9,0 \% \text { polícias } \\
\end{array}$ & $\begin{array}{c}\text { Professores, amigos e mães } \\
\text { percebidos como os mais } \\
\text { úteis e os pais como menos } \\
\text { úteis. } \\
\text { Mães e os pais pressionam } \\
\text { para a vítima terminar a } \\
\text { relação, com } 20,0 \% \text { e } 18,0 \% \\
\end{array}$ & $\begin{array}{l}\text { Invasão de privacidade } \\
\text { Medo de perder o } \\
\text { parceiro }\end{array}$ \\
\hline 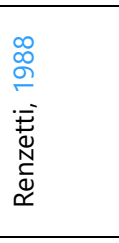 & $\begin{array}{c}\text { Comportamentos } \\
\text { de pedido de } \\
\text { ajuda e eficácia da } \\
\text { atuação das fontes } \\
\text { formais de apoio } \\
\text { E.U.A. }\end{array}$ & $\begin{array}{c}100 \mathrm{M} \\
26-35 \text { anos }\end{array}$ & $\begin{array}{l}\text { Questionário } \\
\text { comportamentos de } \\
\text { pedido de ajuda e a } \\
\text { sua eficácia }\end{array}$ & 78,0 & - & - & $\begin{array}{l}57,0 \% \text { classificaram casas } \\
\text { abrigo, a atuação das polícias } \\
\text { e psicólogos como pouco } \\
\text { eficazes/competentes }\end{array}$ & $\begin{array}{l}\text { Ineficiência dos serviços } \\
\text { de apoio à vítima }\end{array}$ \\
\hline 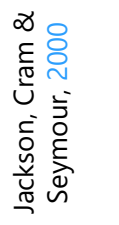 & 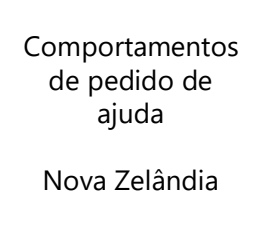 & $\begin{array}{c}373 \text { alunos } \\
16-20 \text { anos } \\
53,6 \% \text { M } 46,4 \% \\
H\end{array}$ & $\begin{array}{l}\text { Questionário de } \\
\text { comportamentos de } \\
\text { ajuda }\end{array}$ & - & $\begin{array}{c}\text { Vítimas violência } \\
\text { sexual }(46,8 \% \mathrm{H} \mathrm{e} \\
46,1 \% \mathrm{M}) \text { ou } \\
\text { violência física } \\
(55,2 \% \mathrm{M} \text { e } 45,5 \% \\
\mathrm{H}) \text { não revelaram }\end{array}$ & - & $\begin{array}{l}\text { Pedido de ajuda percebido } \\
\text { pelos participantes como } \\
\text { sendo benéfico }\end{array}$ & $\begin{array}{l}\text { Medo de represálias e } \\
\text { retaliação }\end{array}$ \\
\hline 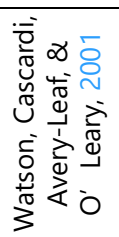 & $\begin{array}{l}\text { Comportamentos } \\
\text { de pedido de } \\
\text { ajuda } \\
\text { E.U.A. }\end{array}$ & $\begin{array}{c}476 \text { alunos do } \\
\text { ensino médio } \\
\text { Média }=16,63 \\
\text { anos } \\
44,0 \% \mathrm{M} \mathrm{e} \\
56,0 \% \mathrm{H}\end{array}$ & $\begin{array}{l}\text { Listas de verificação de } \\
\text { resposta da } \\
\text { vitimização com } 5 \\
\text { categorias }\end{array}$ & - & $\begin{array}{l}\text { Mulheres }(57,0 \%) \\
\text { > Homens }(27,0 \%) ;\end{array}$ & $\begin{array}{l}\text { Apoio informal } \\
(43,0 \%)>\text { apoio } \\
\text { formal }(8,0 \%)\end{array}$ & - & - \\
\hline 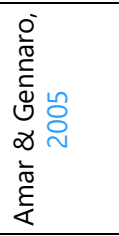 & $\begin{array}{l}\text { Comportamentos } \\
\text { de pedido de } \\
\text { ajuda } \\
\text { E.U.A. }\end{array}$ & $\begin{array}{c}863 \mathrm{M} \\
\text { estudantes } \\
\text { universitárias } \\
18-25 \text { anos }\end{array}$ & $\begin{array}{l}\text { Abuse Assessment } \\
\text { Screen }\end{array}$ & 40,0 & - & $\begin{array}{l}\text { Amigos }(50,0 \%) \\
\text { Familiares }(25,0 \%)\end{array}$ & - & - \\
\hline
\end{tabular}


Tabela 1

Síntese dos Estudos Considerados na Revisão Sistemática (continuação)

\begin{tabular}{|c|c|c|c|c|c|c|c|c|}
\hline \multirow{3}{*}{ 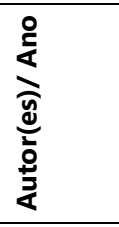 } & \multirow{3}{*}{$\begin{array}{l}\text { Objetivos do } \\
\text { estudo/País }\end{array}$} & \multicolumn{7}{|c|}{ Principais resultados } \\
\hline & & \multirow{2}{*}{$\begin{array}{c}\text { N, idade, sexo: } \\
M=\text { Mulheres } \\
H=\text { Homens }\end{array}$} & \multirow[b]{2}{*}{ Medidas } & \multicolumn{4}{|c|}{ Comportamentos de pedido de ajuda } & \multirow{2}{*}{$\begin{array}{l}\text { Barreiras } \\
\text { à revelação }\end{array}$} \\
\hline & & & & $\%$ & $\begin{array}{c}\text { Diferenças } \\
\text { H e M }\end{array}$ & Fontes & Resposta percebida & \\
\hline 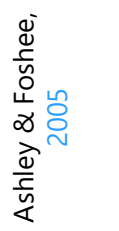 & $\begin{array}{l}\text { Comportamentos de } \\
\text { pedido de ajuda e } \\
\text { fontes utilizadas no } \\
\text { pedido de ajuda } \\
\text { E.U.A. }\end{array}$ & $\begin{array}{l}225 \text { vítimas } \\
\text { adolescentes } \\
14-16 \text { anos } \\
72,0 \% \mathrm{M} \mathrm{e} \\
28,0 \% \mathrm{H}\end{array}$ & $\begin{array}{l}\text { Questionários } \\
\text { elaborados para o } \\
\text { efeito do estudo }\end{array}$ & 50,0 & $\begin{array}{c}\text { Homens }(63,0 \%)> \\
\text { Mulheres }(34,0 \%) \\
\text { apoio formal }\end{array}$ & $\begin{array}{c}\text { Apoio informal } \\
(93,0 \%)>\text { apoio } \\
\text { formal }(40,0 \%) \\
\text { Amigos }(89,0 \%)\end{array}$ & - & - \\
\hline 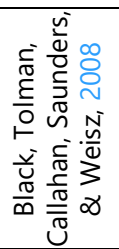 & $\begin{array}{c}\text { Comportamentos de } \\
\text { pedido de ajuda } \\
\text { E.U.A. }\end{array}$ & $\begin{array}{c}57 \text { alunos do } 9 .^{\circ} \\
\text { ao } 12 .^{\circ} \text { anos } \\
56,0 \% \mathrm{M}_{4} 44,0 \% \\
\mathrm{H}\end{array}$ & $\begin{array}{l}\text { Questionário } \\
\text { "Response Options for } \\
\text { Survey Questions" } \\
\text { elaborado para o } \\
\text { efeito do estudo }\end{array}$ & 67,0 & $\begin{array}{c}\text { Mulheres }(78,1 \%)> \\
\text { Homens }(52,0 \%)\end{array}$ & - & - & $\begin{array}{c}\text { Desvalorização da } \\
\text { violência }(75,0 \%) \text { e } \\
\text { naturalização do ciúme } \\
\text { como prova de amor }\end{array}$ \\
\hline 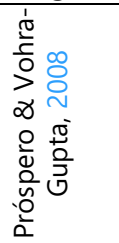 & $\begin{array}{c}\text { Comportamentos de } \\
\text { pedido de ajuda e } \\
\text { fontes utilizadas no } \\
\text { pedido de ajuda } \\
\text { E.U.A. }\end{array}$ & $\begin{array}{c}200 \text { estudantes } \\
\text { universitários } \\
18-25 \text { anos } \\
69,0 \% \mathrm{M} ; 31,0 \% \\
\mathrm{H}\end{array}$ & $\begin{array}{l}\text { Questionário } \\
\text { elaborado para o } \\
\text { efeito do estudo }\end{array}$ & 42,0 & - & $\begin{array}{l}\text { Amigos e familiares } \\
\text { Serviços de saúde } \\
\text { mental }(16,0 \%)\end{array}$ & - & $\begin{array}{c}\text { Vergonha } \\
\text { Despesa financeira } \\
\text { Ineficácia do sistema } \\
\text { Receio de } \\
\text { estigmatização }\end{array}$ \\
\hline 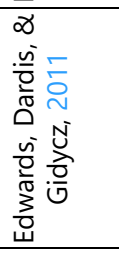 & $\begin{array}{l}\text { Comportamentos de } \\
\text { pedido de ajuda } \\
\text { E.U.A. }\end{array}$ & $\begin{array}{c}107 \mathrm{M} \\
\text { estudantes } \\
\text { universitárias } \\
\mathrm{M}=19 \text { anos }\end{array}$ & $\begin{array}{l}\text { Checklist } \\
\text { Três questões abertas } \\
\text { elaboradas para o } \\
\text { efeito do estudo }\end{array}$ & 75,0 & $73,0 \% \mathrm{M}$ & Amigas & $\begin{array}{l}\text { Amigos como os úteis após } \\
\text { a divulgação }(56,0 \%) \\
\text { Amigos como igualmente } \\
\text { vistos como os menos úteis } \\
\text { de apoio/ajuda }(49,0 \%)\end{array}$ & $\begin{array}{l}\text { Desvalorização da } \\
\text { violência }(80,0 \%)\end{array}$ \\
\hline 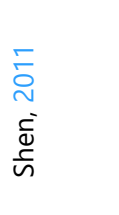 & $\begin{array}{c}\text { Comportamentos de } \\
\text { pedido de ajuda e } \\
\text { barreiras inerentes ao } \\
\text { pedido de ajuda } \\
\text { Taiwan }\end{array}$ & $\begin{array}{c}10 \mathrm{M} \\
20 \text { e } 28 \text { anos }\end{array}$ & $\begin{array}{c}\text { Entrevista } \\
\text { semiestruturada }\end{array}$ & 4,0 & - & - & $\begin{array}{l}\text { Inexistência de apoio } \\
\text { de amigos e família }\end{array}$ & $\begin{array}{c}\text { Não importunar os } \\
\text { outros } \\
\text { Receio de revitimização } \\
\text { da família } \\
\text { Desconhecimento dos } \\
\text { serviços disponíveis }\end{array}$ \\
\hline
\end{tabular}


Tabela 1

Síntese dos Estudos Considerados na Revisão Sistemática (continuação)

\begin{tabular}{|c|c|c|c|c|c|c|c|c|}
\hline \multirow{3}{*}{ 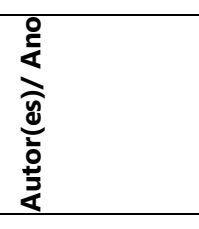 } & \multirow{3}{*}{$\begin{array}{l}\text { Objetivos do } \\
\text { estudo/País }\end{array}$} & \multicolumn{6}{|c|}{ Principais resultados } & \multirow{3}{*}{$\begin{array}{l}\text { Barreiras } \\
\text { à revelação }\end{array}$} \\
\hline & & \multirow{2}{*}{$\begin{array}{c}N, \text { idade, sexo: } \\
M=\text { Mulheres } \\
H=\text { Homens }\end{array}$} & \multirow{2}{*}{ Medidas } & \multicolumn{4}{|c|}{ Comportamentos de pedido de ajuda } & \\
\hline & & & & $\%$ & $\begin{array}{c}\text { Diferenças } \\
\mathrm{H} \text { e M }\end{array}$ & Fontes & $\begin{array}{l}\text { Resposta } \\
\text { percebida }\end{array}$ & \\
\hline 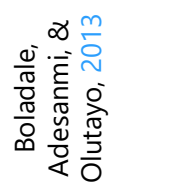 & $\begin{array}{c}\text { Comportamentos de } \\
\text { pedido de ajuda } \\
\text { Nigéria }\end{array}$ & $\begin{array}{c}362 \text { estudantes } \\
18-35 \text { anos } 51,7 \% \\
\text { M; } 48,3 \% \mathrm{H}\end{array}$ & $\begin{array}{l}\text { Questionário } \\
\text { elaborado para o } \\
\text { efeito do estudo }\end{array}$ & $\begin{array}{c}7,4 \\
93,7 \text { não } \\
\text { divulgou }\end{array}$ & - & - & - & $\begin{array}{l}\text { Gravidade da } \\
\text { violência }(5,3 \%)\end{array}$ \\
\hline 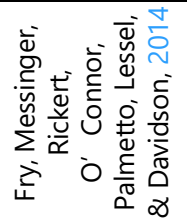 & $\begin{array}{c}\text { Comportamentos de } \\
\text { pedido de ajuda e } \\
\text { identificar barreiras ao } \\
\text { pedido de ajuda } \\
\text { E.U.A. }\end{array}$ & $\begin{array}{c}1.312 \text { alunos do } \\
\text { secundário } \\
14-17 \text { anos } \\
56,0 \% \mathrm{M} ; 44,0 \% \mathrm{H}\end{array}$ & $\begin{array}{l}\text { Questionários } \\
\text { comportamentos de } \\
\text { ajuda elaborados para } \\
\text { o efeito do estudo }\end{array}$ & 61,0 & - & $\begin{array}{l}\text { Apoio informal, junto dos } \\
\text { amigos }(72,0 \%)\end{array}$ & $\begin{array}{l}\text { Abandonar o/a } \\
\text { agressor/a } \\
\text { sugerido por } \\
80.1 \% \text { das } \\
\text { fontes }\end{array}$ & - \\
\hline 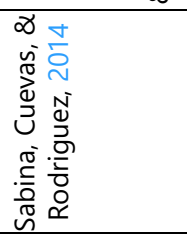 & $\begin{array}{l}\text { Comportamentos de } \\
\text { pedido de ajuda } \\
\text { E.U.A. }\end{array}$ & $\begin{array}{c}1.525 \text { adolescentes } \\
12-18 \text { anos } 50,7 \% \\
M ; 49,3 \% H\end{array}$ & $\begin{array}{l}\text { Questionário } \\
\text { modificado utilizado } \\
\text { no estudo Sexual } \\
\text { Assault between } \\
\text { Latinas (SALAS) }\end{array}$ & 60,5 & $\begin{array}{c}\text { Raparigas }(18,4 \\
\text { vezes) }> \\
\text { Rapazes, de } \\
\text { pedir ajuda } \\
\text { formal }\end{array}$ & $\begin{array}{c}\text { Apoio informal }(60,7 \%)> \\
\text { apoio formal }(15,6 \%)\end{array}$ & - & $\begin{array}{c}\text { Vergonha } \\
\text { Legitimação da } \\
\text { violência }\end{array}$ \\
\hline 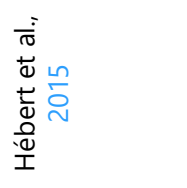 & $\begin{array}{c}\text { Comportamentos de } \\
\text { pedido de ajuda } \\
\text { E.U.A. }\end{array}$ & $\begin{array}{l}8.194 \text { estudantes } \\
14-21 \text { anos }\end{array}$ & $\begin{array}{l}\text { Questionário "Self- } \\
\text { efficacy to Deal with } \\
\text { Violence Scale" }\end{array}$ & - & $\begin{array}{c}\text { Raparigas } \\
(63,1 \%)> \\
\text { Rapazes } \\
(50,6 \%)\end{array}$ & - & - & $\begin{array}{c}\text { Vergonha } \\
\text { Impotência } \\
\text { Baixa } \\
\text { autoestima e } \\
\text { autoeficácia }\end{array}$ \\
\hline 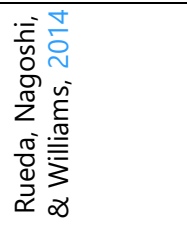 & $\begin{array}{l}\text { Comportamentos de } \\
\text { pedido de ajuda } \\
\text { E.U.A. }\end{array}$ & $\begin{array}{c}64 \text { adolescentes } \\
15-17 \text { anos } \\
62,5 \% \mathrm{M} ; 37,5 \% \mathrm{H}\end{array}$ & $\begin{array}{l}20 \text { Focus group } \\
\text { (divididos por } \\
\text { aculturação e género) }\end{array}$ & - & - & - & $\begin{array}{c}\text { Raparigas são } \\
\text { mais } \\
\text { pressionadas a } \\
\text { deixar a relação } \\
\text { que os rapazes }\end{array}$ & $\begin{array}{l}\text { Medo de } \\
\text { perder o/a } \\
\text { parceiro/a } \\
\text { Medo de } \\
\text { retaliação }\end{array}$ \\
\hline
\end{tabular}




\section{Tabela 1}

Síntese dos Estudos Considerados na Revisão Sistemática (continuação)

\begin{tabular}{|c|c|c|c|c|c|c|c|c|}
\hline \multirow{3}{*}{ 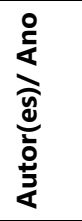 } & \multirow{3}{*}{$\begin{array}{l}\text { Objetivos do } \\
\text { estudo/País }\end{array}$} & \multicolumn{6}{|c|}{ Principais resultados } & \multirow{3}{*}{$\begin{array}{l}\text { Barreiras } \\
\text { à revelação }\end{array}$} \\
\hline & & \multirow{2}{*}{$\begin{array}{c}\mathrm{N} \text {, idade, sexo: } \\
M=\text { Mulheres } \\
\mathbf{H}=\text { Homens }\end{array}$} & \multirow{2}{*}{ Medidas } & \multicolumn{4}{|c|}{ Comportamentos de pedido de ajuda } & \\
\hline & & & & $\%$ & $\begin{array}{c}\text { Diferenças } \\
\text { H e M }\end{array}$ & Fontes & Resposta percebida & \\
\hline 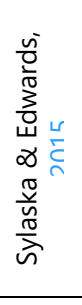 & $\begin{array}{l}\text { Comportamentos de } \\
\text { pedido de ajuda } \\
\text { E.U.A. }\end{array}$ & $\begin{array}{c}391 \text { estudantes } \\
18-25 \text { anos } \\
42,9 \% \mathrm{M} ; 51,9 \% \\
\mathrm{H} \\
75,3 \% \\
\text { homossexual, } \\
19,5 \% \text { queer e } \\
2,6 \% \text { bissexual } \\
\end{array}$ & $\begin{array}{c}\text { Questionário sobre } \\
\text { comportamentos de } \\
\text { ajuda. }\end{array}$ & 35,1 & - & Apoio informal & $\begin{array}{l}\text { 43,0\% das vítimas considerou } \\
\text { o apoio empático como o } \\
\text { mais fornecido pelas fontes. }\end{array}$ & $\begin{array}{l}\text { Relativização da } \\
\text { gravidade da violência } \\
\text { Invasão de privacidade }\end{array}$ \\
\hline 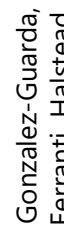 & $\begin{array}{l}\text { Comportamentos de } \\
\text { pedido de ajuda } \\
\text { E.U.A. }\end{array}$ & $\begin{array}{c}11 \mathrm{M} \\
18-24 \text { anos }\end{array}$ & $\begin{array}{c}\text { Entrevista } \\
\text { semiestruturada } \\
\text { elaborada para o efeito. }\end{array}$ & 91,0 & - & - & $\begin{array}{l}\text { Avaliação positiva após } \\
\text { recurso aos serviços formais. }\end{array}$ & $\begin{array}{l}\text { Falta de conhecimento } \\
\text { dos serviços }\end{array}$ \\
\hline 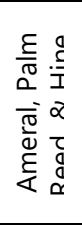 & $\begin{array}{l}\text { Comportamentos de } \\
\text { pedido de ajuda } \\
\text { E.U.A. }\end{array}$ & $\begin{array}{c}4.710 \text { estudantes } \\
\text { universitários } \\
\text { Média idades = } \\
21 \text { anos } \\
70,3 \% \text { M; } 27,2 \% \\
\text { H }\end{array}$ & $\begin{array}{l}\text { Checklist elaborada } \\
\text { para o efeito do estudo }\end{array}$ & 11,8 & $\begin{array}{c}\text { Rapazes < } \\
\text { raparigas }\end{array}$ & - & - & $\begin{array}{l}\text { Não reconhecimento da } \\
\text { violência } \\
\text { Manutenção da } \\
\text { privacidade } \\
\text { Vergonha }\end{array}$ \\
\hline 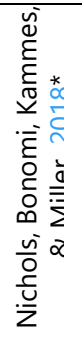 & $\begin{array}{l}\text { Comportamentos de } \\
\text { pedido de ajuda } \\
\text { E.U.A. }\end{array}$ & $\begin{array}{l}27 \text { M estudantes } \\
19-24 \text { anos }\end{array}$ & $\begin{array}{c}\text { Entrevista } \\
\text { semiestruturada }\end{array}$ & 81,5 & - & $\begin{array}{l}\text { Apoio formal: terapia } \\
\text { e aconselhamento } \\
\text { (71,4\%) e serviços } \\
\text { médicos ( } 37,0 \%) \\
\text { 14,8\% polícia } \\
7,3 \% \text { investigação } \\
\text { universitária } \\
\text { 3,7\% advogados }\end{array}$ & - & $\begin{array}{c}\text { Não reconhecer a } \\
\text { violência } \\
\text { Receio estigmatização } \\
\text { Preocupações } \\
\text { financeiras } \\
\text { Precedente experiência } \\
\text { negativa com os serviços } \\
\text { Autoculpabilização da } \\
\text { violência }\end{array}$ \\
\hline
\end{tabular}

Nota. * Estudo apurado no follow-up. 
Caraterísticas amostrais. De acordo com a análise efetuada, o número de participantes envolvidos nos estudos variou entre 10 (Shen, 2011) e 8.194 (Hébert et al., 2015). Os estudos apresentam grande diversidade em termos das características amostrais, seja em termos da idade dos/as participantes, sexo e orientação sexual. Também ao nível da localização geográfica dos estudos se verifica dispersão. $O$ intervalo de idades dos/as participantes situou-se entre os 12 anos (e.g., Sabina et al., 2014) e os 35 anos (e.g., Renzetti, 1988), sendo que oito estudos identificaram uma faixa etária dos 12 aos 18 anos, 11 estudos dos 19 aos 25 anos e cinco estudos apresentaram idades compreendidas entre os 26 e os 35 anos. Seis estudos apontaram como amostra estudantes do ensino médio (e.g., Ashley \& Foshee, 2005; Black et al., 2008) e oito estudos continham uma amostra constituída por estudantes universitários/as (e.g., Amar \& Gennaro, 2005; Ameral et al., 2017; Boladale et al., 2013).

Quanto ao sexo dos/as participantes, verificou-se que em dez estudos o número de participantes de sexo feminino era superior aos de sexo oposto (e.g., Ameral et al., 2017; Ashley \& Foshee, 2005; Próspero \& Vohra-Gupta, 2008). Em sete estudos a amostragem envolvia apenas mulheres (e.g., Amar \& Gennaro, 2005; Edwards et al., 2011; Gonzalez-Guarda et al., 2016). Ademais, somente em dois estudos (Sylaska \& Edwards, 2015; Watson et al., 2001), a percentagem de homens a participar nos estudos era superior à participação feminina. Relativamente à orientação sexual dos/as participantes, o estudo de Renzetti (1988) foi constituído por 100 mulheres lésbicas e no estudo de Sylaska e Edwards (2015) a amostra envolveu 77 vítimas de violência no namoro, sendo que 75,3\% identificou-se como sendo homossexual, $19,5 \%$ queer (pessoas que não seguem o modelo de heterossexualidade ou do binarismo de género) e $2,6 \%$ bissexual. Nos restantes 17 estudos, não foi realizada qualquer menção à orientação sexual dos/as inquiridos/as.

No que diz respeito à localização dos estudos, a grande maioria $(n=16)$ foram realizados nos Estados Unidos da América, um estudo (Boladale et al., 2013) foi realizado no sul da Nigéria, com uma amostra estudantil; outro estudo (Jackson et al., 2000) envolveu 304 adolescentes de cinco escolas secundárias da Nova Zelândia e, por último, um outro (Shen, 2011) que envolveu uma amostra de 10 mulheres, com idades entre os 20 e 28 anos.

Medidas usadas. Em termos de medidas usadas, a Escala Tática de Conflitos Revisada (The Revised Conflict Tactics Scales - CTS-R) de Murray Straus et al. (1996) surgiu mencionada em cinco estudos (e.g., Ameral et al., 2017; Black et al., 2008; Boladale et al., 2013). Sylaska e Edwards (2015) utilizaram apenas a subescala de agressão física da CTS-R que avaliou as experiências dos participantes sobre as suas relações de namoro. No estudo de Watson et al. (2001) foi administrada a Escala Tática de Conflito Modificada (MCTS), uma versão modificada da Escala Tática de Conflito (CTS) de Straus et al. (1996) que permitiu a inclusão de testemunhos de relacionamentos atuais e com duração inferior ao critério da CTS de 12 meses.

O Inventário de Conflitos nas Relações de Namoro entre Adolescentes (CADRI) foi administrado nos estudos de Hébert et al. (2015) e Rueda et al. (2014) como forma de averiguar a vitimização dos adolescentes inquiridos. Em quatro estudos a vitimização foi verificada através de entrevistas ou questionários a jovens (e.g., Ashley \& Foshee, 2005; Fry et al., 2014).

\section{Principais resultados dos estudos.}

Comportamentos de pedido de ajuda: indicadores. Em relação à adoção de algum tipo de comportamentos de pedido de ajuda, um número considerável de estudos $(n=9)$ (e.g., Amar \& Gennaro, 2005; Black et al., 2008; Edwards et al., 2011), registou considerações de pedidos de ajuda por parte das vítimas, situamse estas entre um mínimo de 40,0\% (Amar \& Gennaro, 2005) e de máximo de 92,0\% (Mahlstedt \& Keeny, 1993). Em contrapartida, em oito estudos verificou-se também uma tendência para a não denúncia (e.g., Ameral et al., 2017; Ashley \& Foshee, 2005; Boladale et al., 2013; Jackson et al., 2000). Assim, no estudo de Boladale et al. (2013) 92,6\% dos/as estudantes universitários/as vítimas de violência nas suas relações amorosas não denunciaram a violência sofrida e apenas 7,4\% pediram ajuda. Também Ashley e Foshee (2005) verificaram que das 225 vítimas inquiridas somente 90 pediram ajuda. Esta reduzida expressão do 
pedido de ajuda foi também corroborada pelo estudo de Sylaska e Edwards (2015), em que apenas um terço das 77 vítimas, na sua maioria homossexuais, revelaram os abusos na relação de intimidade. No estudo de Jackson et al. (2000), que aborda o pedido de ajuda nas diferentes tipologias abusivas, verificou-se que perante a vitimização por violência física e sexual as vítimas tendem a não denunciar estes abusos. No entanto Fry et al. (2014) encontraram uma taxa de pedido de ajuda superior a $60,0 \%$ nos casos de vitimação por violência física e/ou sexual.

Comportamentos de pedido de ajuda: diferenças de género. Relativamente às diferenças de género ao nível dos comportamentos de pedido de ajuda foi registada uma maior tendência para as mulheres pedirem ajuda comparativamente com os homens $(n=6)$ (e.g., Ameral et al., 2017; Black et al., 2008; Edwards et al., 2011; Hébert et al., 2015). Os homens surgiram como sendo mais propensos a não adotar qualquer tipo de ação e assumiram que abordam menos o tema ou consideram mesmo que não necessitam de efetuar tal abordagem. Em anuência ao referido, as percentagens de pedidos de ajuda por parte de homens situavam-se entre um mínimo de 27,0\% (Watson et al., 2001) e um máximo de 63,0\% (Ashley \& Foshee, 2005). Já o pedido de ajuda por parte das mulheres oscilou, consoante os estudos, entre os $46,8 \%$ (Jackson et al., 2000) e os 78,1\% (Black et al., 2008). No estudo de Hébert et al. (2015), comparou-se a prestação de ajuda por fontes de divulgação que presenciassem a violência com o pedido de ajuda das vítimas e verificou-se que o número de oferta de ajuda (16,4\% mulheres vs. $15,1 \%$ homens) foi superior ao número de pedido de ajuda (14,9\% mulheres vs. $14,0 \%$ homens), revelando-se as mulheres mais propensas a oferecer e a pedir ajuda em situações de violência no namoro.

Comportamentos de pedido de ajuda: fontes. Quanto às fontes a quem foi realizado o pedido de ajuda, dois estudos (Edwards et al., 2011; Mahlstedt \& Keeny, 1993) comprovaram que as vítimas de violência no namoro revelaram a situação abusiva a pelo menos uma fonte. Nos dez estudos que abordaram as fontes de divulgação perante uma situação de violência no namoro, a divulgação informal surgiu como tendo sido a mais adotada por vítimas de violência no namoro $(n=9)$ (e.g., Amar \& Gennaro, 2005; Ashley \& Foshee, 2005; Edwards et al., 2011). No estudo de Watson et al. (2001) 43,0\% dos/as participantes revelaram recorrer a ajuda informal, seguido de terminar o relacionamento abusivo (37,0\%), depois de resposta agressiva (35,0\%), a opção de não ter qualquer tipo de ação violenta (32,0\%) e por último 8,0\% das vítimas relataram recorrer a ajuda formal. Ao nível das fontes de divulgação informal, as vítimas revelaram preferência no pedido de ajuda a amigos, seguido de irmã/o(s) e pai(s)/mãe(s) num número considerável de estudos ( $n=6$ ) (e.g., Amar \& Gennaro, 2005; Ashley \& Foshee, 2005; Edwards et al., 2011). No estudo de Fry et al. (2014) foi também clara a preferência de divulgação a amigas, com $72,0 \%$ das vítimas a pedir ajuda junto destas. Na divulgação formal, a fonte de revelação mais escolhida pelas vítimas de violência no namoro foram os/as agentes educativos/as ou terapeutas em cinco estudos (e.g., Amar \& Gennaro, 2005; Ashley \& Foshee, 2005; Edwards et al., 2011), a polícia em quatro estudos (Ashley \& Foshee, 2005; Boladale et al., 2013; Mahlstedt \& Keeny, 1993; Sabina et al., 2014; Sylaska \& Edwards, 2015) e professores/as em três estudos (Ashley \& Foshee, 2005; Mahlstedt \& Keeny, 1993; Sylaska \& Edwards, 2015). Segundo dados de Ashley e Foshee (2005), os homens eram mais propensos a pedir ajuda formal do que as mulheres (63,0\% e 34,0\%, respetivamente). Contrariamente ao referido, as mulheres mostravam um aumento de 18,33 vezes nas probabilidades de pedir ajuda formal quando comparadas aos homens (Sabina et al., 2014).

Comportamentos de pedido de ajuda: resposta percebida. A família e os amigos surgiram, em seis estudos (e.g., Edwards et al., 2011; Fry et al., 2014; Jackson et al., 2000), identificados pelas vítimas de violência no namoro como sendo os mais prováveis a oferecer um apoio adequado. Porém, em três estudos (Edwards et al., 2011; Renzetti, 1988; Shen, 2011), os/as amigos/as também foram considerados/as os/as menos úteis, dada a maior probabilidade de efetuar julgamentos ou provocar aborrecimentos. Shen (2011) verificou no seu estudo que a ajuda destes era muitas vezes inapropriada, indiferente ou limitada a apoio emocional ou material; os/as amigos/as e a família tendiam a atribuir a responsabilidade à vítima e a promoveram a autossuficiência desta para lidar com a situação abusiva. Dos seis estudos que abordaram 
os tipos de apoios fornecidos pelas fontes às vítimas de violência no namoro, quatro apresentaram como tipo de ajuda mais oferecido e percebido como sendo mais útil, o apoio sentimental, ações como ouvir e conversar com a vítima e fornecer conselhos (Edwards et al., 2011; Fry et al., 2014; Jackson et al., 2000; Sylaska \& Edwards, 2015). Concomitantemente, em três estudos (e.g., Edwards et al., 2011; Jackson et al., 2000; Sylaska \& Edwards, 2015) foram identificados comportamentos em que as fontes de divulgação não compreendiam e ridicularizavam a situação e tentavam assumir o controlo da situação. Em outros três estudos, as fontes de revelação abordaram diretamente o/a agressor/a da vítima, exigindo uma mudança de comportamento (Fry et al., 2014; Rueda et al., 2014; Sylaska \& Edwards, 2015). O incentivo ao término da relação foi um comportamento registado em seis estudos (e.g., Edwards et al., 2011; Fry et al., 2014; Jackson et al., 2000). No estudo de Mahlstedt e Keeny (1993), a mãe e o pai surgiram percebidos como os que mais pressionam para o fim do relacionamento $(30,0 \%$ e $18,0 \%$, respetivamente). Em quatro estudos (Fry et al., 2014; Jackson et al., 2000; Rueda et al., 2014; Watson et al., 2001) as mulheres surgiram como sendo as mais pressionadas a abandonar a relação abusiva e a acabar efetivamente por terminar o relacionamento abusivo comparativamente com o sexo oposto. Por fim, em três estudos (Jackson et al., 2000; Rueda et al., 2014; Watson et al., 2001) as vítimas de sexo masculino optaram por não adotar qualquer atitude resolutiva depois do pedido de ajuda.

Barreiras ao pedido de ajuda. De entre as principais barreiras listadas nesta análise que tendiam a bloquear e/ou inibir a ação das vítimas, identificou-se: i) legitimação da violência e menorização da violência ( $n=7$ ) (e.g., Ameral et al., 2017; Black et al., 2008; Boladale et al., 2013; Edwards et al., 2011); ii) vergonha $(n=5)$ (e.g., Ameral et al., 2017; Hébert et al., 2015; Nichols et al., 2018); iii) sentimento exacerbado e medo de perder o/a parceiro/a $(n=4)$; (e.g., Jackson et al., 2000; Mahlstedt \& Keeny, 1993); iv) autossuficiência para resolver a situação $(n=3)$ (Boladale et al., 2013; Gonzalez-Guarda et al., 2016; Shen, 2011); v) ausência de conhecimento acerca dos serviços disponíveis ( $n=3$ ) (Boladale et al., 2013; Gonzalez-Guarda et al., 2016; Shen, 2011), vii) evasão da privacidade ( $n=3$ ) (Ameral et al., 2017; Mahlstedt \& Keeny, 1993; Sylaska \& Edwards, 2015) e viii) o medo de represálias e retaliação por parte do/a parceiro/a violento/a após uma possível divulgação das vítimas $(n=2)$ (Jackson et al., 2000; Rueda et al., 2014). De entre os motivos mais apontados pelas vítimas de violência no namoro para o não pedido de ajuda junto de profissionais de saúde destacou-se: o facto de estes serem percecionados como sendo dispendiosos $(n=2)$ (Nichols et al., 2018; Próspero \& Vohra-Gupta, 2008); a perceção da inutilidade daqueles e o facto de recearem serem percebidos como doentes (Próspero \& Vohra-Gupta, 2008), ou, ainda, o facto de temerem ser rotulados como vítimas de violência por parte do/a parceiro/a e a existência de anterior experiência negativa com este tipo de serviços (Nichols et al., 2018). Por fim, no estudo de Renzetti (1988), os/as participantes mencionaram que os serviços de apoio a vítimas não estão preparados para atender situações que envolvam relações homossexuais e as suas especificidades.

\section{Discussão}

A presente revisão sistemática centrou-se na análise de 19 estudos que focaram os comportamentos efetivos de pedido de ajuda por parte de vítimas de violência no namoro, excluindo investigações que abordassem intenções e ações indiretas de pedido de ajuda e/ou revelação da experiência íntima abusiva.

A análise dos 19 estudos permitiu concluir pela não consensualidade quanto à tendência de revelação ou não por parte das vítimas de violência no namoro, sendo que o pedido de ajuda parece oscilar entre os $78,0 \%$, apurado no estudo de Renzetti (1988) conduzido com uma amostra de 100 mulheres, nos Estados Unidos da América, com idades compreendidas entre os 26 e os 35 anos e os $11,8 \%$ no estudo de Ameral et al. (2017), com uma amostra de 4.710 estudantes universitários/as na sua maioria mulheres, média de idade 21 anos e também realizado nos Estados Unidos da América. Relativamente ao sexo das fontes disponíveis para prestar ajuda em situações de violência no namoro, as mulheres foram identificadas 
como sendo as mais propensas a prestar ajuda comparativamente com o sexo oposto (Hébert et al., 2015).

No que diz respeito à faixa etária das vítimas de violência no namoro, verificou-se que nos estudos com uma amostra com idades superiores a 18 anos $(n=13)$, em sete destes estudos uma grande parte das vítimas admitiu priorizar o silêncio (e.g., Amar \& Gennaro, 2005; Shen, 2011; Sylaska \& Edward, 2015). Não obstante, foi possível observar nos diferentes estudos, uma maior tendência por parte das participantes de sexo feminino em admitir o pedido de ajuda $(n=6)$ para fazer face à situação de vitimização na intimidade (e.g., Ameral et al., 2017; Black et al., 2008; Hébert et al., 2015) comparativamente com o sexo oposto. De igual modo, as mulheres admitiram, nos estudos, maior probabilidade de se separarem do/a agressor/a (Watson et al., 2001), sendo que foram também as mulheres aquelas que relataram maiores indicadores de vitimização. Por sua vez, os participantes de sexo masculino surgiram como sendo menos propensos a responderem a itens de pedido de ajuda quando comparados com as mulheres, apresentando uma certa inação perante a violência na medida em que não procuram revelar a situação violenta ou mesmo cessar a relação abusiva. Além disso, os homens que mantêm relações com outra pessoa do mesmo sexo aumentam o estigma social que inibe o pedido de ajuda (Sabina et al., 2014). Segundo Allen, Ridgeway e Swan (2015), as barreiras à divulgação são mais acentuadas quando o problema é percecionado como sendo não-normativo na sociedade, logo esta inércia poderá ser melhor compreendida se atendermos a certas representações ideológicas associadas aos homens e as quais tendem a interferir ou inibir a sua capacidade de pedido de ajuda e de reação à vitimização em geral (Fry et al., 2014; Watson et al., 2001). Ademais, foram vários os estudos (e.g., Jackson et al., 2000; Lavoie, Vézina, Piché, \& Boivin, 1995) que concluíram que os homens tendem a minimizar e a tolerar mais a violência do que as mulheres. Tais evidências revelam-se algo preocupantes e devem ser devidamente consideradas nas políticas de prevenção e intervenção no fenómeno da violência das relações de intimidade.

A legitimação da violência foi a barreira mais apontada pelos/as participantes dos estudos $(n=7)$ (e.g., Ameral et al., 2017; Black et al., 2008; Boladale et al., 2013; Edwards et al., 2011); em que os/as jovens consideraram a agressão não gravosa ("não era nada sério"). Não raras as vezes os/as jovens podem ser confrontados com situações relacionais, pautadas por comportamentos violentos e outras formas de coerção, face aos quais poderão ser levados a adotar uma postura de legitimação, percecionando tais atos como uma manifestação de amor e/ou ciúme (e.g., Black et al., 2008; Caridade \& Machado, 2013). Adicionalmente tem sido documentado que as vítimas tendem a resolver as situações de vitimação sozinhas - cultura de autossuficiência $(n=3)$ - seja por vergonha de se assumirem como vítimas, seja por temerem perder a sua privacidade ou mesmo por não quererem importunar a família e amigos com assuntos desta natureza (Shen, 2011). O desconhecimento dos meios de ajuda formal existentes surgiu identificada como uma outra importante barreira para a procura de ajuda por parte das vítimas $(n=3)$, algo que se afigura deveras preocupante dado que as instituições de apoio às vítimas são aquelas que se revelam qualificadas para prestar o devido suporte e apoio à vítima (Black et al., 2008; Fry et al., 2014).

As fontes de revelação informais surgiram como sendo as mais usadas pelas vítimas e de forma mais específica os/as amigos/as ( $n=9$ ) (e.g., Black et al., 2008; Boladale et al., 2013; Edwards et al., 2011; Rueda et al., 2014). Os pares de sexo feminino foram identificados pelas vítimas como os mais procurados (Edwards et al., 2011; Fry et al., 2014), ainda que os de sexo masculino surjam percebidos como os mais prováveis de manterem a informação em segredo (Jackson et al., 2000).

Concomitantemente, e no que respeita ao tipo de orientação mais fornecida às vítimas pelos pares $(n=$ 3) envolve o aconselhar o pedido de ajuda junto de um adulto ou falar diretamente com este, considerando-os como sendo as fontes com mais poder para intervir caso a violência escale (Fry et al., 2014; Jackson et al., 2000; Mahlstedt \& Keeny, 1993). Todavia, como indicação menos frequente a dar às vítimas $(n=3)$ verificou-se a sugestão de recorrer a serviços formais de ajuda (Amar \& Gennaro, 2005; Fry et al., 2014; Próspero \& Vohra-Gupta, 2008). Denota-se, deste modo, que parece existir uma certa desconfiança/insegurança relativamente ao recurso aos sistemas formais de apoio, motivada 
fundamentalmente por fatores de privacidade, aplicação correta das leis e julgamentos que podem advir da divulgação, que não raras vezes, promovem uma revitimização (Nichols et al., 2018). A vitimização secundária é especialmente emergente quando estão presentes nestes/as profissionais, crenças e atitudes erróneas que potenciam a invalidação da experiência da vítima, acabando por se constituir como barreiras à denúncia (Machado et al., 2009). Também Sabina et al. (2014) referem que os/as jovens tendem a não recorrer a serviços formais por receio de proibição do contacto com o/a parceiro/a e para não expor toda a situação abusiva sofrida aos pais e envolver estes em todo o processo. As vítimas referiram que tendem a rejeitar o pedido de ajuda nas fontes de divulgação formal como os/as agentes educativos/as, órgãos de polícia criminal e professores/as, priorizando os/as amigos/as como os/as mais prováveis a oferecer bom apoio $(n=5)$ (Amar \& Gennaro, 2005; Ashley \& Foshee, 2005; Mahlstedt \& Keeny, 1993; Rueda et al., 2014; Sylaska \& Edwards, 2015). Não obstante, os/as jovens não estão habilitados para ajudar os outros perante uma situação de violência no namoro, podem ter medo do/a agressor/a do amigo ou até podem responsabilizar a vítima (Jackson et al., 2000; Sabina et al., 2014).

\section{Conclusões}

O presente estudo forneceu uma revisão abrangente e sistemática de 19 estudos sobre o pedido de ajuda em situações de violência no namoro, permitindo, deste modo, extrair importantes conclusões. Primeiramente, os principais objetivos dos estudos foram analisar o comportamento de pedido de ajuda, as fontes de revelação utilizadas e/ou as barreiras inerentes à divulgação da experiência abusiva por parte de vítimas de violência no namoro. Os estudos abordaram estes objetivos de uma forma diferente e nem sempre com a mesma metodologia, levando a resultados marcadamente variáveis. Neste sentido, seria interessante investir em futuras investigações apenas com uma metodologia, de modo a providenciar resultados mais uniformes. Mais especificamente, foi percetível uma grande dispersão entre as taxas de divulgação da violência no namoro, possivelmente motivada pelos diferentes critérios adotados em cada estudo.

Não obstante, importa também referir que a presente revisão sistemática assentou em critérios e tomadas de decisão que condicionam a generalização de conclusões. De referir, desde logo, que se trata de um estudo teórico delimitado a um período temporal, e focado numa temática amplamente estudada. Além disso, apenas uma minoria dos estudos $(n=2)$ contemplados na presente revisão sistemática usaram amostras não heterossexuais, algo que seria de explorar em estudos futuros. A não inclusão de estudos não publicados (dissertações e relatórios de pesquisa) pode influenciar os resultados obtidos e também é possível que alguns estudos relevantes, pese embora o recurso a amostragem por bola de neve, não tenham emergido na pesquisa efetuada.

A informação sistematizada no presente trabalho pode, é nossa expectativa, desempenhar um contributo importante na formação dos/as profissionais que trabalham com vítimas de violência nas relações de namoro. Um conhecimento mais estruturado acerca dos motivos que levam os/as jovens a não procurar ajuda e a permanecer na relação abusiva, pode assumir um papel central na proteção das vítimas, nomeadamente na adoção de ações que facilitem a saída segura e permanente da relação abusiva, como por exemplo a indicação de um abrigo para vítimas onde estas podem permanecer de forma a garantir a sua segurança. Na presente revisão sistemática, os amigos surgiram identificados como as fontes preferenciais de revelação, sendo certo que estes se revelam, não raras vezes, imaturos e que não possuem conhecimentos suficientes para prestar um adequado suporte e apoio em situações de violência no namoro. Torna-se, deste modo, crucial que as iniciativas em matéria de prevenção e intervenção continuem a sensibilizar os/as jovens para estas temáticas, de forma a precaver casos de banalização da violência e permanência na relação abusiva aquando o pedido de ajuda junto dos pares.

Conflito de interesses | Conflict of interest: nenhum | none.

Fontes de financiamento | Funding sources: nenhuma | none.

Contributos: IP: Revisão da literatura; Tratamento e discussão dos dados; Redação do manuscrito. SC: Tratamento e discussão dos dados; Redação e revisões do manuscrito. 


\section{Referências}

Allen, C. T., Ridgeway, R., \& Swan, S. C. (2015). College students' beliefs regarding help seeking for male and female sexual assault survivors: Even less support for male survivors. Journal of Aggression, Maltreatment \& Trauma, 24(1), 102-115. https://doi.org/10.1080/10926771.2015.982237

Amar, A. F., \& Gennaro, S. (2005). Dating violence in college women: Associated physical injury, healthcare usage, and mental health symptoms. Nursing Research, 54(4), 235-242. https://doi.org/10.1097/00006199200507000-00005

Ameral, V., Palm Reed, K. M., \& Hine, D. A. (2017). An analysis of help seeking patterns among college student victims of sexual assault, dating violence, and stalking. Journal of Interpersonal Violence, 2017, 1-25. https://doi.org/10.1177/0886260517721169

Ashley, O. S., \& Foshee, V. A. (2005). Adolescent help-seeking for dating violence: Prevalence, sociodemographic correlates, and sources of help. Journal of Adolescent Health, 36(1), 25-31. https://doi.org/10.1016/j.jadohealth.2003.12.014

Black, B. M., Tolman, R. M., Callahan, M., Saunders, D. G., \& Weisz, A. N. (2008). When will adolescents tell someone about dating violence victimization? Violence Against Women, 14(7), 741-758. https://doi.org/10.1177/1077801208320248

Boladale, M., Adesanmi, A., \& Olutayo, A. (2013). Experience of childhood violence and help-seeking behaviour of students exposed to dating violence at the Obafemi Awolowo University, Ile-Ife. Gender \& Behaviour, 21(2), 277-289.

Caridade, S. (2011). Vivências íntimas violentas: Uma abordagem científica [Violent intimate experiences: A scientific approach]. Coimbra: Edições Almedina.

Caridade, S. (2018). Violência no namoro: Contextualização teórica e empírica [Dating violence: Theoretical and empirical contextualization]. In S. Neves \& A. Correia (Coord.), Violências no Namoro (pp. 9-40). Maia: Edições ISMAI.

Caridade, S., \& Barros, S. (2018). Violência nas relações íntimas juvenis e ideação e comportamentos suicidas [Dating violence, ideation and suicidal behaviors]. Psicologia, Saúde \& Doenças, 19(2), 323-336. https://doi.org/10.15309/18psd190213

Caridade, S., \& Machado, C. (2013). Violência nas relações íntimas juvenis: Uma revisão da investigação, prática e teoria [Violence in juvenile intimate relations: A review of research, practice, and theory]. Psicologia, 27(1), 91-113. Retrieved from http://www.scielo.mec.pt/pdf/psi/v27n1/v27n1a06.pdf

Caridade, S., Pinheiro, I., \& Dinis, A. (2019). Disclosure in victims of dating violence: Strategies and reasons for help-seeking. In W. Spencer (Ed.), Dating violence: Prevalence, risk factors and perspectives (pp. 85-106). New York: Nova Science Publishers, Inc.

Carter-Snell, C. (2015). Youth dating violence: A silent epidemic. In M. Taylor, \& J. A. Pooley (Eds.), Overcoming domestic violence (pp. 49-65). New York: Nova Science Publishers.

Centers for Disease Control and Prevention (CDC). (2012). Understanding teen dating violence: Fact sheet. Retrieved from https://www.cdc.gov/violenceprevention/pdf/teen-dating-violence-2014-a.pdf Center16

Centers for Disease Control and Prevention (CDC). (2016). Understanding teen dating violence. Fact sheet. 2016. Retrieved from http://www.cdc.gov/violenceprevention/pdf/teen-dating-violencefactsheet-a.pdf

Dixe, M., Rodrigues, A., Freire, C., Rodrigues, G., Fernandes, M., \& Dias, T. (2010). A violência de género na relação de namoro em estudantes do ensino superior: Práticas e comportamentos de violência [Gender violence in dating relationship in higher education students: Practices and behaviors of violence]. Comunicação apresentada no Sétimo Simpósio Nacional de Investigação em Psicologia, Braga. Retrieved from https://iconline.ipleiria.pt/handle/10400.8/334

Edwards, K. M., Dardis, C. M., \& Gidycz, C. A. (2011). Women's disclosure of dating violence: A mixed methodological study. Feminism \& Psychology, 22(4), 507-517. https://doi.org/10.1177/0959353511422280

Exner-Cortens, D., Eckenrode, J., \& Rothman, E. (2013). Longitudinal associations between teen dating violence victimization and adverse health outcomes. Pediatrics, 131(1), 71-78. https://doi.org/10.1542/peds.20121029

Foshee, V. A., Reyes, H. L. M., Gottfredson, N. C., Chang, L.-Y., \& Ennett, S. T. (2013). A longitudinal examination of psychological, behavioral, academic, and relationship consequences of dating abuse victimization among 
a primarily rural sample of adolescents. Journal of Adolescent Health, 53(6), 723-729. https://doi.org/10.1016/j.jadohealth.2013.06.016

Fry, D. A., Messinger, A. M., Rickert, V. I., O'Connor, M. K., Palmetto, N., Lessel, H., \& Davidson, L. L. (2014). Adolescent relationship violence: Help-seeking and help-giving behaviors among peers. Journal of Urban Health, 91(2), 320-334. https://doi.org/10.1007/s11524-013-9826-7

Gonzalez-Guarda, R. M., Ferranti, D., Halstead, V., \& Ilias, V. M. (2016). Experiences with dating violence and help seeking among Hispanic females in their late adolescence. Issues in Mental Health Nursing, 37(4), 229 238. https//doi.org/10.3109/01612840.2016.1141341

Guerreiro, A., Teixeira, A. M., Dias, A., Pontedeira, C., Cordeiro, J., Magalhães, M. J., ... Mendes, T. (2017). Violência no namoro: Resultados nacionais apontam a gravidade do problema [Dating violence: National results point to severity of problems]. UMAR 2017. Porto: União de Mulheres Alternativa e Reposta. Retrieved

from http://www.umarfeminismos.org/images/stories/noticias/Relatorio_de_Imprensa_Final.pdf

Hamby, S., \& Turner, H. (2013). Measuring teen dating violence in males and females: Insights from the national survey of children's exposure to violence. Psychology of Violence, 3(4), 323-339. https//doi.org/10.1037/a0029706

Hébert, M., Van Camp, T., Lavoie, F., Blais, M., \& Guerrier, M. (2015). Understanding the hesitancy to disclose teen dating violence: Correlates of self-efficacy to deal with teen dating violence. Victimology Society of Serbia, 17(4), 43-64. https://doi.org/10.2298/TEM1404043H

Hedge, J. M., Sianko, N., \& McDonell, J. R. (2017). Professional help-seeking for adolescent dating violence in the rural south: The role of social support and informal help-seeking. Violence Against Women, 23(12), 14421461. https://doi.org/10.1177/1077801216662342

Jackson, S. M., Cram, F., \& Seymour, F. W. (2000). Violence and sexual coercion in high school students' dating relationships. Journal of Family Violence, 15(1), 23-36. https://doi.org/10.1023/A:1007545302987

Jennings, W. G., Okeem, C., Piquero, A. R., Sellers, C. S., Theobald, D., \& Farrington, D. P. (2017). Dating and intimate partner violence among young persons ages 15-30: Evidence from a systematic review. Aggression and Violent Behavior, 33, 107-125. https://doi.org/10.1016/j.avb.2017.01.007

Lavoie, F., Vézina, L., Piché, C., \& Boivin, M. (1995). Evaluation of a prevention program for violence in teen dating relationships. Journal of Interpersonal Violence, 10(4), 516-524. https://doi.org/10.1177/088626095010004009

Machado, C., Caridade, S., \& Martins, C. (2010). Violence in juvenile dating relationships self-reported prevalence and attitudes in a Portuguese sample. Journal of Family Violence, 25(1), 43-52. https://doi.org/10.1007/s10896-009-9268-x

Machado, C., Matos, M., Saavedra, R., Cruz, O., Antunes, C., Pereira, M., ... Capitão, L. (2009). Crenças e atitudes dos profissionais face à violência conjugal: Estudos com profissionais de saúde, polícias e professores [Beliefs and attitudes of professionals about marital violence: Studies with health professionals, policemen and teachers]. Acta Médica Portuguesa, 22(6), 735-742. Retrieved from https://www.actamedicaportuguesa.com/revista/index.php/amp/article/view/1739/1318

Mahlstedt, D., \& Keeny, L. (1993). Female survivors of dating violence and their social networks. Feminism \& Psychology, 3(3), 319-333. https://doi.org/10.1177/0959353593033003

Moher, D., Liberati, A., Tetzlaff, J., Altman, D. G., \& PRISMA Group. (2009). Preferred reporting items for systematic reviews and meta-analyses: The PRISMA Statement. PLoS Medicine, 6(7), e1000097. https://doi.org/10.1371/journal.pmed.1000097

Neves, A. S., Cameira, M., Machado, M., Duarte, V., \& Machado, F. (2016). Beliefs on marital violence and selfreported dating violence: A comparative study of Cape Verdean and Portuguese adolescents. Journal of Child \& Adolescent Trauma, 11(2), 197-204. https://doi.org/10.1007/s40653-016-0099-7

Nichols, E. M., Bonomi, A., Kammes, R., \& Miller, E. (2018). Service seeking experiences of college-aged sexual and intimate partner violence victims with a mental health and/or behavioral disability. Journal of American College Health, 66(6), 487-495. https://doi.org/10.1080/07448481.2018.1440572

Organização Mundial de Saúde (OMS). (2016). Violence against women: Intimate partner violence and sexual violence against women. Retrieved from http://www.who.int/mediacentre/factsheets/fs239/en/

Próspero, M., \& Vohra-Gupta, S. (2008). The use of mental health services among victims of partner violence on college campuses. Journal of Aggression, Maltreatment \& Trauma, 16(4), 376-390. https://doi.org/10.1080/10926770801926450 
Renzetti, C. M. (1988). Violence in gay and lesbian domestic partnerships. New York: Harrington Park.

Rueda, H. A., Nagoshi, J. L., \& Williams, L. R. (2014). Mexican American and European American adolescents' dating experiences across the ecosystem: Implications for healthy relationships within an ecodevelopmental framework. Journal of Human Behavior in the Social Environment, 24(3), 358-376. https://doi.org/10.1080/10911359.2013.831013

Sabina, C., Cuevas, C. A., \& Rodriguez, R. M. (2014). Who to turn to? Help-seeking in response to teen dating violence among Latinos. Psychology of Violence, 4(3), 348-362. https://doi.org/10.1037/a0035037

Sabina, C., \& Ho, L. Y. (2014). Campus and college victim responses to sexual assault and dating violence: Disclosure, service utilization, and service provision. Trauma, Violence, \& Abuse, 15(3), 201-226. https://doi.org/10.1177/1524838014521322

Santos, A. P., Caridade, S., \& Cardoso, J. (2019). Violência nas relações íntimas juvenis: (Des)ajustamento psicossocial e estratégias de coping [Violence in juvenile intimate relationships: psychosocial (des)adjustment and coping strategies]. Contextos Clínicos, 12(1) 1-25. https://doi.org/1010.4013/ctc.2019.121.01

Shen, A. C.-T. (2011). Cultural barriers to help-seeking among Taiwanese female victims of dating violence. Journal of Interpersonal Violence, 26(7), 1343-1365. https://doi.org/10.1177/0886260510369130

Soares, J., Lopes, M., \& Njaine, K. (2013). Violência nos relacionamentos afetivo-sexuais entre adolescentes de Porto Alegre, Rio Grande do Sul, Brasil: Busca de ajuda e rede de apoio [Violence in affective and sexual relationships among adolescents in Porto Alegre, Rio Grande do Sul State, Brazil: Search for help and support networks]. Cadernos de Saúde Pública, 29(6), 1121-1130. https://doi.org/10.1590/S0102311X2013001000009

Straus, M. A. (2004). Prevalence of violence against dating partners by male and female university students worldwide. Violence Against Women, 10(7), 790-811. https://doi.org/10.1177/1077801204265552

Straus, M. A., Hamby, S. L., Boney-McCoy, S., \& Sugarman, D. (1996). The revised conflict tactics scales (CTS2): Development and preliminary psychometric data. Journal of Family Issues, 17(3), 283-316. https://doi.org/10.1177/019251396017003001

Sylaska, K. M., \& Edwards, K. M. (2015). Disclosure experiences of sexual minority college student victims of intimate partner violence. American Journal of Community Psychology, 55(3-4), 326-335. https://doi.org/10.1007/s10464-015-9717-z

Watson, J. M., Cascardi, M., Avery-Leaf, S., \& O'Leary, K. D. (2001). High school students' responses to dating aggression. Violence and Victims, 16(3), 339-348. https://doi.org/10.1891/0886-6708.16.3.339

Whitlock, J., Wyman, P. A., \& Moore, S. R. (2014). Connectedness and suicide prevention in adolescents: Pathways and implications. Suicide and Life-Threatening Behavior, 44(3), 246-272. https://doi.org/10.1111/sltb.12071 